\title{
The Toxoplasma Tour de Force to Unfold its Intravacuolar Developmental Program
}

\author{
Georgios Pavlou, Isabelle Tardieux* \\ Institute for Advanced Biosciences (IAB), Team Membrane dynamics of parasite-host cell interactions, CNRS UMR5309, INSERM U1209, Université Grenoble \\ Alpes, Grenoble, France
}

\section{Article Info}

\section{Article Notes}

Received: September 10, 2018

Accepted: October 20, 2018

\section{*Correspondence:}

Dr. Isabelle Tardieux, Institute for Advanced Biosciences (IAB), Team Membrane dynamics of parasite-host cell interactions, CNRS UMR5309, INSERM U1209, Université

Grenoble Alpes, Grenoble, France;

Email: isabelle.tardieux@inserm.fr

C 2018 Tardieux I. This article is distributed under the terms of the Creative Commons Attribution 4.0 International License.

\section{Keywords:}

Toxoplasma gondii

Invasive nano-device

Host cell Invasion

Membrane fission

Dynamins

Vacuole biogenesis

Original Article Citation: Pavlou G, Biesaga M, Touquet B, Lagal V, Balland M, Dufour A, Hakimi MA, Tardieux I. Toxoplasma Parasite Twisting Motion Mechanically Induces Host Cell Membrane Fission to Complete Invasion within a Protective Vacuole. Cell Host Microbe. 2018 Jul 11;24(1):8196.e5. doi: 10.1016/j.chom.2018.06.003. Epub 2018 Jun 28. PMID:30008293

\section{Abstract}

Toxoplasma gondii is an obligate intracellular single-celled eukaryotic parasite with an impressive ability to invade virtually all nucleated cells from all warm-blooded animals, within a second time-scale. The invasive T. gondii tachyzoite achieves this feat by injecting a multi-unit nanodevice in the plasma membrane and underlying cortical cytoskeleton of the targeted cell that serves as an anchor point to withstand the parasite invasive force. Whether this nanodevice could also contribute at the latest step of invasion when the budding entry vesicle pinches off of the plasma membrane as a parasitophorous vacuole had not been yet addressed. Using fluorescent versions of both a parasite nanodevice component and a reporter for the target plasma membrane in conjunction with quantitative high-resolution live imaging, Pavlou et al. characterized the nanodevice toroidal shape once inserted in the membrane as well as its stretching and shrinking when accommodating the passage of the several micron-sized ellipsoid shaped tachyzoite. Tracking in real time the motion of internal eccentric markers allowed defining the tachyzoite final rotation along the long axis which imposes a twisting motion on its basal pole and directs closure of the torus hence promoting both sealing and release of the entry vesicle. Monitoring distinct host cell plasma markers allowed Pavlou et al. to propose that the twisting motion could also act as an initial mechanical trigger for the transition to the intracellular lifestyle. Their publication therefore brings evidence for a key new contribution of the nanodevice to end the high-speed multi-step invasion process.

\section{Introduction}

The protozoan Toxoplasma gondii belongs to the phylum of Apicomplexa that exceeds 6000 members, most of which depend on hosting cells to achieve their life cycle ${ }^{1,2}$. T. gondii is considered as one of the most successful Apicomplexa because of its worldwide distribution, its host spectrum that comprises most warm-blooded vertebrates, human included, and its amazing ability to colonize virtually any type of nucleated cells in only a few tens of seconds. The T. gondii developmental stage called tachyzoite is a model of choice for investigating the mechanisms of cell invasion because this polarized cell has high invasive and replicative skills and is tractable to genetic modifications owing to recent conceptual and technological advances in molecular genetics ${ }^{3,4,5}$. About a decade ago, the discovery of a protein complex stored in the neck of apical secretory organelles typifying the phylum, namely the rhoptries, has been a major breakthrough in the Toxoplasma-cell invasion field of investigation. This protein complex is first released at the tachyzoite apical tip and injected into the target cell surface - Plasma Membrane (PM) and cortex - after a still molecularly undefined interaction. Made of four RhOptry Neck proteins - RON2, 4, 5, 8 - 
and defined as the RON complex ${ }^{6,7}$, this complex is seen as the main component of the Zoite-Cell circular Junction (ZCJ) often named Tight Junction (TJ) that bridges the two cells during the invasion process $\mathrm{s}^{8,9,10}$. While in the 1980-1990s, the ZCJ was described as an electron dense close contact or a moving junction for several species of zoites invading their respective host cells, recent live imaging on T. gondii (strain RH type 1) tachyzoites that express a fluorescent tagged version of RON2 - which spans the target cell PM - allowed demonstrating the anchor point function of the ZCJ. This platform was indeed shown to be anchored to the target cell cortex ${ }^{11}$ and withstand a tachyzoite actin myosin (myosinAmyoA)-based traction force ${ }^{12,13}$ driving the parasite into a budding Entry Vesicle (EV). At about the same time genetic evidence raised the possibility that the traction force model does not fully explain the motile and invasive potential of tachyzoites. Motor-independent mechanisms, in particular relying on viscoelastic properties of the tachyzoite cell were first proposed ${ }^{14,15}$. Then a compensatory function by myoC for the genetic loss of myoA has been claimed ${ }^{16}$, but real time imaging of $\Delta$ myoA parasites argues rather for an alternative mode of entry depending on the host cell actin when the parasite is unable to apply its own traction force on the $\mathrm{ZCJ}{ }^{17}$.

During uptake by non-phagocytic and phagocytic host cells, a majority of intracellular microbes subvert the endocytosis pathway ${ }^{18,19}$. The process that promotes scission of endocytic buds (or pits) from the cell PM accounts for the endosome birth and involves the activity of ubiquitous mechano-enzymes that belong to the dynamin family. Dynamins assemble as helical oligomers wrapping around the neck of budding vesicles and applying torsional and contractile forces on the two membranes apposed at the neck region hence directing membrane fission ${ }^{20,21}$. Interestingly, early electrophysiology studies on tachyzoite entry into cells highlighted shared features with the endocytic fusion pore during endosome formation ${ }^{22}$ while pharmacological and in situ imaging assays suggested the functional contribution of host cell dynamins during the release of the tachyzoite-loaded $\mathrm{EV}^{23,24}$. However, in absence of direct proof for such contribution and because of the recent identification of off target effects for dynamin inhibitors, Pavlou et al. decided to examine the features of the RON complex at the time of closure and immediately post-closure. Their working hypothesis was that the RON complex positioned at the interface between the host cell $\mathrm{PM}$ and the budding EV could influence the process leading to the pinch off of the vacuole-containing tachyzoite, namely the Parasitophorous Vacuole (PV). Their main findings are discussed below.

The twisting motion of the T. gondii tachyzoite from the ZCJ platform, coincides with the RON torus closure

An important limitation in dissecting the multi-step sequence that defines a successful entry event relates to the second-scale dynamics of the process, hence difficult to accurately characterize. To provide the best framework for assessing the invasive skills of the parasite, the Tardieux laboratory has in the recent past engineered a T. gondii line (RH type I strain) in which the gene encoding RON2 is replaced by a chimeric sequence encoding a fluorescent RON2-mCherry (RON2-mC) as well as human cell lines expressing a set of constructs to target the GFP protein at the cell $\mathrm{PM}^{13}$. Using spinning-disk laser scanning live microscopy with high spatio-temporal resolution, Pavlou et al. could resolve the early secretion of the RON2-mCcontaining complex that organizes a toroidal ZCJ platform, which progressively stretches and shrinks as the ellipsoidal zoite passes through and forces the budding of an EV. More excitingly, they observed that once the tachyzoite basal end is about to reach the torus, the parasite executes an anticlockwise rotation that coincides with closure of the torus (Figure 1). The closed status of the torus was convincingly assessed by the disconnection of any extra material tightly bound posteriorly on the invading tachyzoite occurring concomitantly with the zoite twisting motion. Next, the definition of a long axis with the two RON$\mathrm{mC}$ signals from the rhoptries at the apex and the closed torus at the basal pole respectively, together with the detection of a set of internal eccentric fluorescent spherical markers, allowed automatic "xyt" tracking of the three particles. This unambiguously identified a rotation of the internal marker around the long axis, a rotation imposed by the twisting motion of the tachyzoite. In addition, when the tracking was coupled with the analysis of the signal intensity of the internal particle, it was possible to detect periodic fluctuations in pixel intensity which agrees with a continuous rotation of the internal marker, hence of the parasite. Moreover, several lines of evidence suggest that the host cell cortical tension and contractibility underneath the torus could significantly determine the number of $360^{\circ}$ rotation of the internal particle around the axis, and therefore the number of parasite twists "required" prior to PV release.

\section{$\mathrm{PV}$ release requires the parasite twisting motion but not the major host cell membrane fission players namely dynamins and Alix}

The authors interrogated whether the key host cell membrane fission players, namely dynamins, could assist the process of PV release. Quantifying and monitoring invasion in MEF cells genetically silenced for the three dynamin encoding-genes ${ }^{25}$ they found that dynamins are not required for full invasiveness of tachyzoites unlike previously thought. Importantly in the mutant cells lacking dynamins, the invasion scenario is identical with those in dynamin-expressing cells and includes the typical twisting motion prior to the pinch off of the PM that terminates 
A
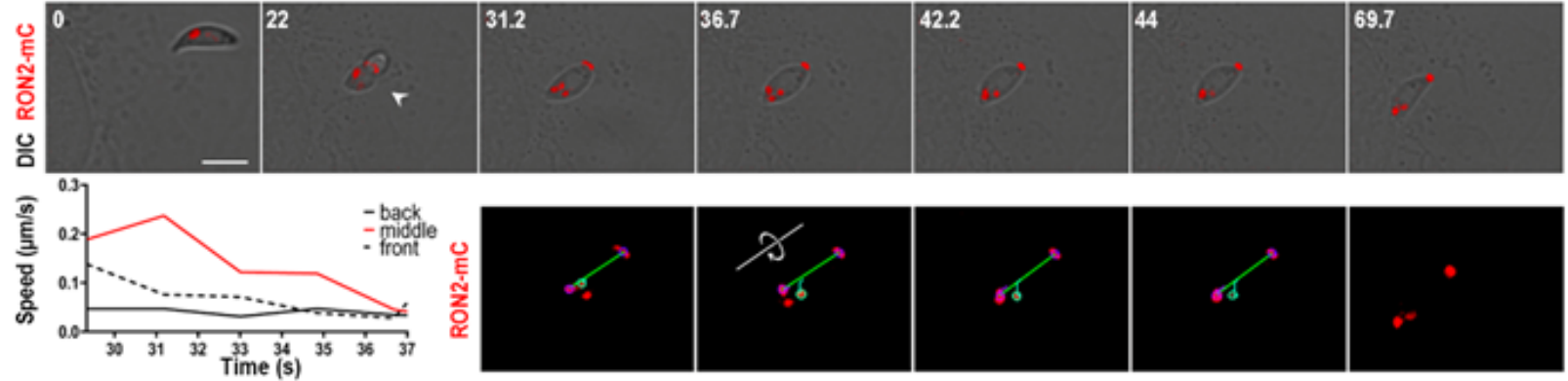

B
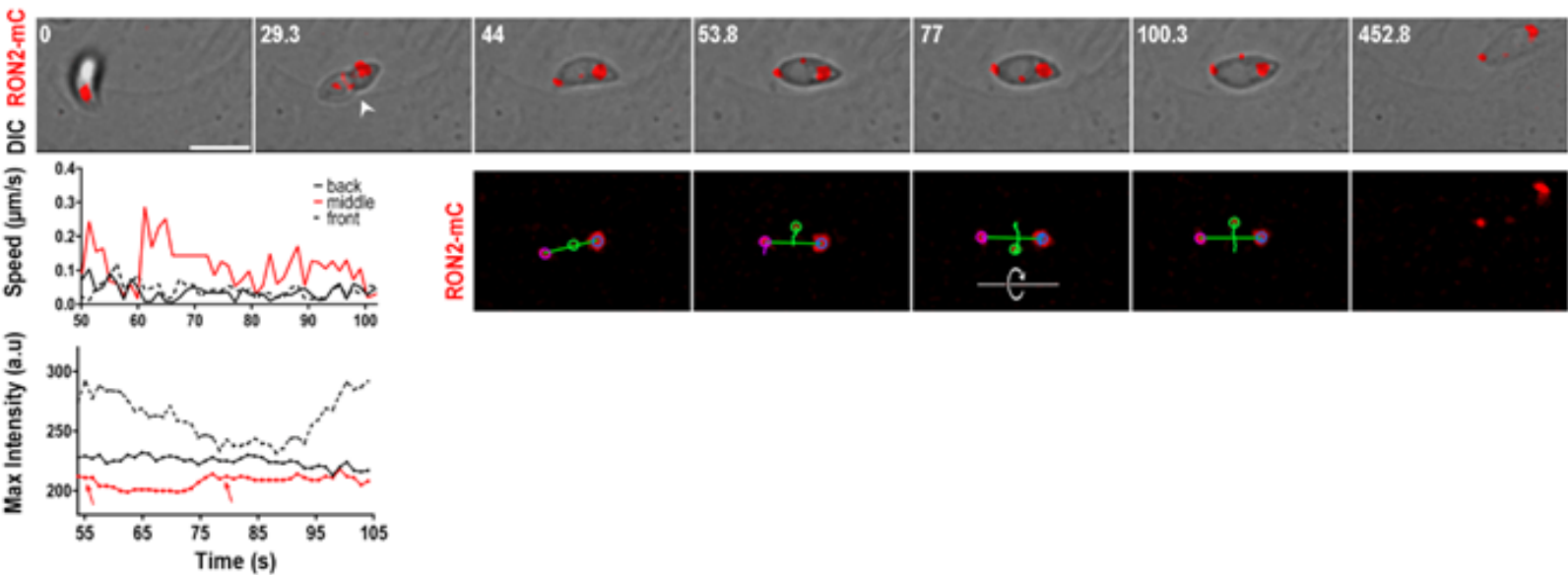

Figure 1: The twisting motion of the $T$. gondii tachyzoite from the ZCJ platform, coincides with the RON torus closure

(A, B) Time lapses of DIC (top) and fluorescent channel overlays (bottom) show the parasites prior to RON2-mC release and halfway inside a U2OS (b) or a $\operatorname{MEF}(\mathrm{c}$ ) cell (see the torus marked by arrowheads). Upon torus closure, automatic tracking of the RON2-mC pools at the parasite poles is reported by blue circles and short blue trajectories while the cytoplasmic RON2-mC circular object (middle, green circle) tracking identifies the twisting motion ( $180^{\circ}$ for $b, \sim 540^{\circ}$ for $c$ ) of the parasite along its main axis (green bar between the two poles, see the circular arrows). Graphs (A, top B) indicate the speed of the three objects tracked and bottom B shows the signal intensity profile of the tracked objects. Note (i) the higher speed of the internal reference and (ii) the periodic fluctuations in signal intensity that correlates with the position with regard to focal point (full focus is indicated by red arrows).

the process. Besides the dynamin machinery, the Endosomal Sorting Complexes Required for Transport (ESCRT) family of proteins has also been characterized as driving membrane scission. The ESCRTs, in particular the ESCRT-III known to form helical polymers and their cofactors, control the release of enveloped viral particles in the extracellular milieu, the cytokinetic abscission of mammalian cells as well as the biogenesis of multivesicular bodies ${ }^{26,27}$. Since the ESCRT-III cofactor Alix has been detected at the ZCJ during cell invasion ${ }^{28}$, the authors directly assessed whether the lack of Alix in cells derived from $\Delta$ Alix mice could impact the PV scission process. In disagreement with this study, they found no quantitative or qualitative difference of the whole tachyzoite cell invasion process in Alix-positive or -deficient cells. Importantly while the tachyzoite applies torque on the RON torus independently of host cell dynamins, Pavlou et al. do not exclude that dynamin partners in particular actin or other host cell component could collaborate with the twisting motion to drive membrane fission upstream the torus.
Closure of the torus is required for PV biogenesis and could serve as an initiating mechanical signal for transition to unfold and complete $T$. gondii developmental program

To functionally correlate the tachyzoite twisting motion with the mechanism of torus closure, Pavlou et al. designed an innovative assay in which the invading tachyzoite carries microbeads at the basal pole, which makes it unable to properly close the torus at the ZCJ. Under this "artificial" situation, while they did not observe twisting motion, they monitored the unusual inflow of extracellular sodium ions that progressively filled the budding EV space. The osmotic unbalance between the bud and the host cell led to a balloon-like expansion of the former and the death of the tachyzoite, presumably due to an increased pressure in the balloon (Figure 2). Interestingly, based on the fluorescence stability of the balloon membrane, the authors concluded that despite the ZCJ site showed leakiness, the ZCJ preserved 
A

A

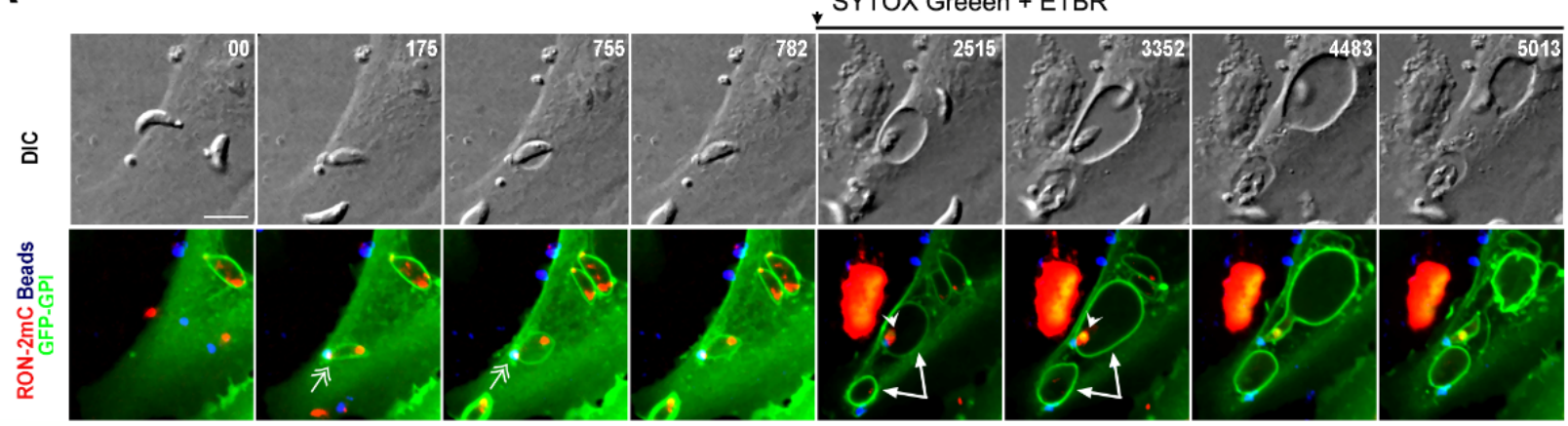

B

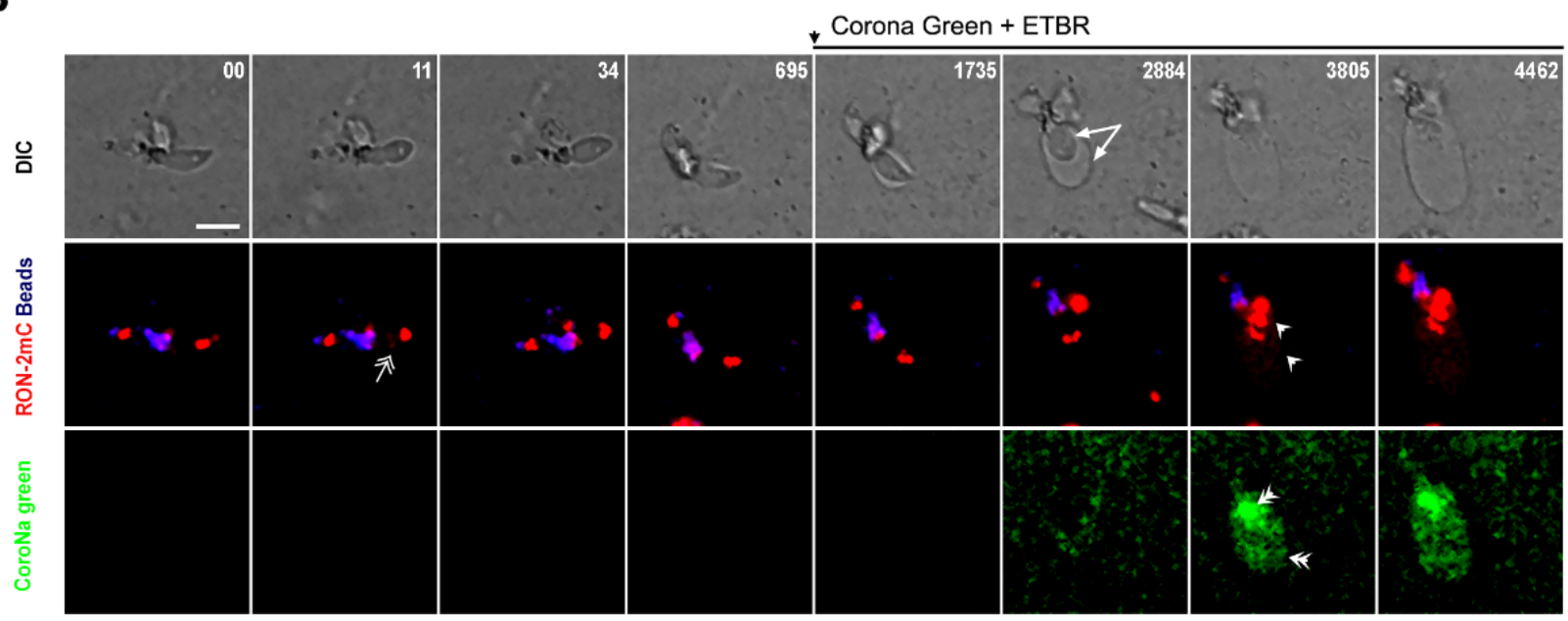

Figure 2: Closure of the torus is required for PV biogenesis and mechanical impairment of the process causes parasite death due to osmotic damages

(A, B) Time lapses show DIC (top) and fluorescent channel overlays (lower). Tachyzoites attached to microbeads (blue) and to both a bead and a partner (B). (A) At about 40 min post-block, SYTOX green and ETBR added to the extracellular milieu (as indicated with an arrow above the frame) are readily incorporated in the cell debris in the medium and are detected in the tachyzoite nucleus indicating loss of membrane integrity (arrowheads). Note that the extracellular beads are surrounded by PM protrusions and with time the huge balloon (arrows) can separate from the parasite to undergo vesiculation. (B) At about 45-60 min post-block, ETBR (arrowheads) and Corona green reagents (double arrowheads) are detected in the PV and the parasite, attesting torus leakiness and subsequent tachyzoite death.

its ability to sieve the molecular components of the PM entering into the composition of the enlarging bud. Indeed, if the balloon had resulted from the stretching of the initial bud membrane, a proportional loss of fluorescence would have characterized the balloon membrane. In contrast changes in the membrane fluorescence can be visualized in the unborn PV immediately post twisting motion and post torus closure. These changes reveal that when the parasite has lost contact with the extracellular milieu - i.e., the EV is sealed - lipids and proteins composing the membrane of the EV ready to pinch already reassemble as new microdomains. This dynamic feature of the PVM suggests import or/and removal of material signing for the early maturation of the PV niche.

\section{Conclusion}

Benefiting from recent advances in both live imaging that provide significant gain in spatio-temporal resolution and T.gondii molecular genetics that eases the introduction of fluorescent markers in the genome, Pavlou et al. have collected highly informative images on the invasion process. More specifically, image processing with open source and home made algorithms provided an accurate qualitative and quantitative description of the tachyzoite behavior at the end of the process (see Graphical abstract, Figure 3). Data analysis led the authors uncovering a mechanism by which the extracellular parasite actively accesses the intracellular environment within a cell PMderived bud. While the nano-scale architecture of the RON torus within the recipient PM in the course of entry remains puzzling and deserves to be investigated in depth, this work unveiled a new functional contribution of the RON complex. Qualifying this complex as invasive nanodevice, these authors document that a parasite-derived torque applied on the nanodevice can indeed account for proper sealing and subsequent release of a membrane-bound PV 


\section{Birth of $T$. gondii tachyzoite intracellular vacuole}
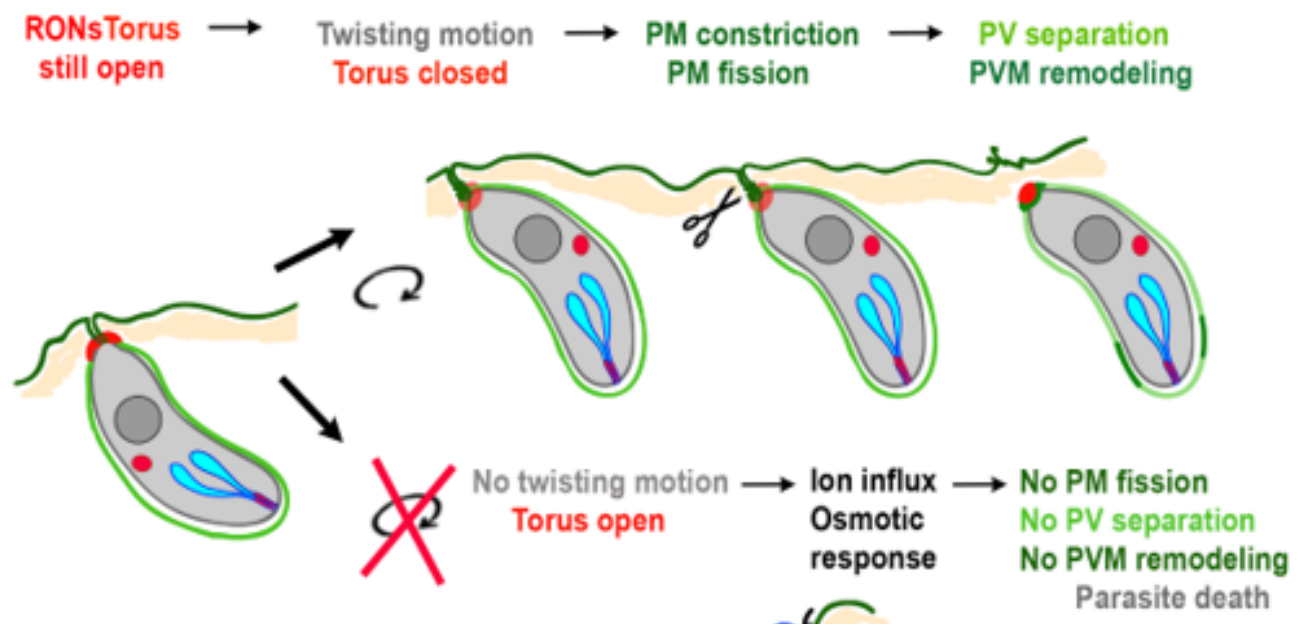

\section{Birth of endosome during CME}

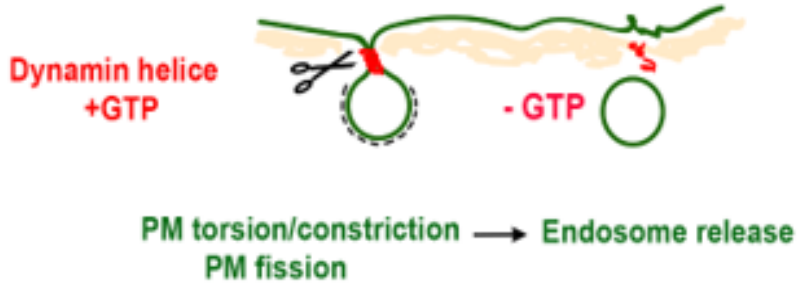

Figure 3: Schematic representation of the host cell invasion process by $T$. gondii.

The invasive tachyzoite stage injects an invasive nanodevice into the Plasma Membrane (PM) of the target cell that organises as a torus and promotes the formation of a tight Zoite-Cell Junction (ZCJ). The nanodevice successively stretches and shrinks while the parasite enters within a budding entry vesicle derived from the PM. Closure of the nanodevice and vesicle sealing behind the parasite are critical for the release of the parasite protective vacuole in the host cell cytoplasm. This study unveils a twisting motion of the tachyzoite imposing rotation on its basal end and likely constriction/torsion of the entry vesicle neck that facilitates membrane fusion and fission upstream the torus to release a Parasitophorous Vacuole (PV) whose membrane (PVM) is rapidly remodelled. When closure is mechanically prevented by microbeads that stay tightly bound to the posterior end of the tachyzoite, the leaky ZCJ causes an osmotic response from the host cell leading to parasite lysis. This work suggests a functional similarity between the mode of action of (i) the GTPase dynamins that form helices around the neck of vesicles (endocytic buds) at the PM to apply torsion and constriction forces that mediate fission (i.e. release of endosomes) and (ii) the torque applied by the tachyzoite on the toroidal nanodevice to ensure PV birth and intracellular development.

while preserving the host cell PM integrity. In addition, the authors monitored for the first time how dynamic is the PV compartment: whether the early remodeling contributes to protect the PV from host cell endocytic processing and build a niche where to expand a replicating-prone tachyzoite progeny is the next question to address. This study also leads to ask fascinating follow-up questions on the forces underlying the torque and the identity of host cell factors other than dynamins that could possibly assist the process. Of note, a common requirement for membrane fission under different topologies is the need for a contractile ring and spiral around adjacent membranes which is provided 
by helical polymers of proteins such as dynamins or ESCRTIII $^{21,29}$. Intriguingly the protozoan Toxoplasma has evolved a torus positioned at the interface between the bud neck and the donor PM, which is twisted and constricted immediately prior to membrane rupture upstream.

\section{References}

1. Gubbels MJ, Duraisingh MT. Evolution of apicomplexan secretory organelles. Int J Parasitol. 2012; 42: 1071-1081.

2. Seeber F, Steinfelder S. Recent advances in understanding apicomplexan parasites. F1000Res. 2016; 5.

3. Huynh MH, Carruthers VB. Tagging of endogenous genes in a Toxoplasma gondii strain lacking Ku80. Eukaryotic Cell. 2009; 8: 530-539.

4. Fox BA, Ristuccia JG, Gigley JP, et al. Efficient gene replacements in Toxoplasma gondii strains deficient for nonhomologous end joining. Eukaryotic Cell. 2009; 8: 520-529.

5. Sidik SM, Huet D, Lourido S. CRISPR-Cas9-based genome-wide screening of Toxoplasma gondii. Nat Protoc. 2018; 13: 307-323.

6. Besteiro S, Michelin A, Poncet J, et al. Export of a Toxoplasma gondii rhoptry neck protein complex at the host cell membrane to form the moving junction during invasion. PLoS Pathog. 2009; 5: e1000309.

7. Straub KW, Peng ED, Hajagos BE, et al. The moving junction protein RON8 facilitates firm attachment and host cell invasion in Toxoplasma gondii. PLoS Pathog. 2011; 7: e1002007.

8. Bannister LH, Butcher GA, Dennis ED, et al. Structure and invasive behaviour of Plasmodium knowlesi merozoites in vitro. Parasitology. 1975; 71: 483-491.

9. Aikawa M, Miller LH, Johnson J, et al. Erythrocyte entry by malarial parasites. A moving junction between erythrocyte and parasite. J Cell Biol. 1978; 77: 72-82.

10. Dubey JP, Lindsay DS, Speer CA. Structures of Toxoplasma gondii tachyzoites, bradyzoites, and sporozoites and biology and development of tissue cysts. Clin Microbiol Rev. 1998; 11: 267-299.

11. Gonzalez V, Combe A, David V, et al. Host cell entry by apicomplexa parasites requires actin polymerization in the host cell. Cell Host Microbe. 2009; 5: 259-272.

12. Sibley LD. How apicomplexan parasites move in and out of cells. Curr Opin Biotechnol. 2010; 21: 592-598.

13. Bichet $\mathrm{M}$, Joly $\mathrm{C}$, Henni $\mathrm{AH}$, et al. The toxoplasma-host cell junction is anchored to the cell cortex to sustain parasite invasive force. BMC Biol. 2014; 12: 773.

14. Egarter S, Andenmatten N, Jackson AJ, et al. The toxoplasma ActoMyoA motor complex is important but not essential for gliding motility and host cell invasion. PLoS ONE. 2014; 9: e91819.
15. Whitelaw JA, Latorre-Barragan F, Gras S, et al. Surface attachment, promoted by the actomyosin system of Toxoplasma gondii is important for efficient gliding motility and invasion. BMC Biol. 2017; 15: 1 .

16. Frénal K, Marq JB, Jacot D, . Plasticity between MyoC- and MyoAglideosomes: an example of functional compensation in Toxoplasma gondii invasion. PLoS Pathog. 2014; 10: e1004504.

17. Bichet $\mathrm{M}$, Touquet B, Gonzalez V, et al. Genetic impairment of parasite myosin motors uncovers the contribution of host cell membrane dynamics to Toxoplasma invasion forces. BMC Biol. 2016; 14: 97.

18. Cossart P, Helenius A. Endocytosis of viruses and bacteria. Cold Spring Harb Perspect Biol. 2014; 6.

19. Colonne PM, Winchell CG, Voth DE. Hijacking Host Cell Highways: Manipulation of the Host Actin Cytoskeleton by Obligate Intracellular Bacterial Pathogens. Front Cell Infect Microbiol. 2016; 6: 107.

20. Antonny B, Burd C, De Camilli P, et al. Membrane fission by dynamin: what we know and what we need to know. EMBO J. 2016; 35: 22702284.

21. Colom A, Redondo-Morata L, Chiaruttini N, et al. Dynamic remodeling of the dynamin helix during membrane constriction. Proc Natl Acad Sci USA. 2017; 114: 5449-5454.

22. Suss-Toby E, Zimmerberg J, Ward GE. Toxoplasma invasion: the parasitophorous vacuole is formed from host cell plasma membrane and pinches off via a fission pore. Proc Natl Acad Sci USA. 1996; 93: 8413-8418.

23. Caldas LA, Attias M, de Souza W.. Dynamin inhibitor impairs Toxoplasma gondii invasion. FEMS Microbiol Lett. 2009; 301: 103108.

24. Caldas LA, Soares LL, Henrique Seabra S, et al. Monitoring of dynamin during the Toxoplasma gondii cell cycle. Pathog Dis. 2016; 74.

25. Park RJ, Shen H, Liu L, et al. Dynamin triple knockout cells reveal off target effects of commonly used dynamin inhibitors. J Cell Sci. 2013; 126: 5305-5312.

26. Chiaruttini N, Redondo-Morata L, Colom A, et al. Relaxation of Loaded ESCRT-III Spiral Springs Drives Membrane Deformation. Cell. 2015; 163: 866-879.

27. Votteler J, Sundquist WI. Virus Budding and the ESCRT Pathway. Cell Host Microbe. 2013; 14.

28. Guérin A, Corrales RM, Parker ML, et al. Efficient invasion by Toxoplasma depends on the subversion of host protein networks. Nat Microbiol. 2017; 2: 1358-1366.

29. Lafaurie-Janvore J, Maiuri P, Wang I, et al. ESCRT-III assembly and cytokinetic abscission are induced by tension release in the intercellular bridge. Science. 2013; 339: 1625-1629. 